\title{
Absorption Efficiency and Physical Bounds on Antennas
}

\author{
Mats Gustafsson, ${ }^{1}$ Marius Cismasu, ${ }^{1}$ and Sven Nordebo ${ }^{2}$ \\ ${ }^{1}$ Department of Electrical and Information Technology, Lund University, Box 118, 22100 Lund, Sweden \\ ${ }^{2}$ School of Mathematics and Systems Engineering, Linnaeus University, 35195 Växjö, Sweden
}

Correspondence should be addressed to Marius Cismasu, marius.cismasu@eit.lth.se

Received 1 March 2010; Accepted 21 June 2010

Academic Editor: Heinrich Foltz

Copyright (C) 2010 Mats Gustafsson et al. This is an open access article distributed under the Creative Commons Attribution License, which permits unrestricted use, distribution, and reproduction in any medium, provided the original work is properly cited.

The all spectrum absorption efficiency appears in the physical bounds on antennas expressed in the polarizability dyadics. Here, it is shown that this generalized absorption efficiency is close to $1 / 2$ for small idealized dipole antennas and for antennas with a dominant resonance in their absorption. Also, the usefulness of this parameter is analyzed for estimation of antenna performance. The results are illustrated with numerical data for several antennas.

\section{Introduction}

A new set of physical bounds on antennas was introduced in [1-5]. These bounds relate the performance of the antenna to the electro- and magnetostatic polarizability dyadics of a circumscribing geometry. This generalizes the classical bounds by Chu [6] for spherical geometries to geometries of arbitrary shape. The new bounds are valid for lossless and linearly polarized [1-4] and elliptically polarized [5] antennas. Moreover, the approach can be used to estimate the performances of many small antennas if the polarizabilities of the antennas are used instead of the circumscribing geometries [2-4].

The only parameter in the bound that depends on the dynamic properties of the antenna is the generalized (or all spectrum) absorption efficiency, $\eta$. This is the generalization of the frequency-dependent absorption efficiency analyzed in [7] given by integration of the absorbed and total power, independently, over all wavelengths.

In [1-4], it is demonstrated that $\eta$ is close to $1 / 2$ for many small antennas that are connected to a frequency independent resistive load and matched at their first resonance. This is motivated by the minimum scattering property that small-matched antennas often possess, that is, they scatter as much power as they absorb at the resonance frequency giving an absorption efficiency of $1 / 2$ at the resonance frequency $[7,8]$. Here, it is shown that small idealized dipole antennas with a dominant first single resonance have an all spectrum absorption efficiency $\eta \lesssim 1 / 2$. The region around the resonance is minimum scattering but the contributions from regions away from the resonance scatter slightly more power than is absorbed giving a generalized (all spectrum) absorption efficiency close to but less than $1 / 2$.

Minimum scattering is a property that many non electrically small resonant antennas also possess. Numerical simulation results of common antennas, both electrically small and not small, verify the theoretical results.

\section{Absorption Efficiency}

The physical bounds analyzed in [1-4] are derived for single port, linearly polarized, reciprocal, and lossless antennas with the reflection coefficient $\Gamma(k)$ and the directivity $D(k ; \hat{\mathbf{k}}, \widehat{\mathbf{e}})$, where $k$ denotes the free-space wavenumber, $\widehat{\mathbf{k}}$ the direction, and $\hat{\mathbf{e}}$ the electric polarization. The forward scattering sum rule [1] gives the antenna identity

$$
\begin{aligned}
\int_{0}^{\infty} & \frac{\left(1-|\Gamma(k)|^{2}\right) D(k ; \hat{\mathbf{k}}, \hat{\mathbf{e}})}{k^{4}} \mathrm{~d} k \\
\quad & =\frac{\eta}{2}\left(\hat{\mathbf{e}} \cdot \boldsymbol{\gamma}_{\mathrm{e}} \cdot \hat{\mathbf{e}}+(\hat{\mathbf{k}} \times \hat{\mathbf{e}}) \cdot \boldsymbol{\gamma}_{\mathrm{m}} \cdot(\hat{\mathbf{k}} \times \hat{\mathbf{e}})\right),
\end{aligned}
$$

where $\gamma_{\mathrm{e}}$ and $\gamma_{\mathrm{m}}$ are the electro- and magnetostatic polarizability dyadics, respectively. The integral (1) is bounded in various ways to produce bounds for different applications, 
for example, resonant and constant partial-realized gain in $[1,3,4]$ and ultra-wide band cases in [2]. The resonant case is applicable for antennas with a dominant first single resonance [3]. It is given by

$$
\frac{D(k ; \hat{\mathbf{k}}, \hat{\mathbf{e}})}{Q} \leq \frac{\eta k_{0}^{3}}{2 \pi}\left(\hat{\mathbf{e}} \cdot \boldsymbol{\gamma}_{\mathrm{e}} \cdot \hat{\mathbf{e}}+(\widehat{\mathbf{k}} \times \hat{\mathbf{e}}) \cdot \boldsymbol{\gamma}_{\mathrm{m}} \cdot(\widehat{\mathbf{k}} \times \hat{\mathbf{e}})\right),
$$

where $k_{0}$ is the resonance wavenumber and $Q$ denotes the $Q$-factor at the resonance, that is, it has the half-power fractional bandwidth $B \approx 2 / Q$.

The polarizability dyadics in the right-hand sides of (1) and (2) are easily determined for the antenna or, as an upper bound, for an arbitrary circumscribing geometry ( http://www.mathworks.fr/matlabcentral/fileexchange/ 26806-antennaq) by the solution of the corresponding electro- and magnetostatic equations [1-4]. This leaves the generalized absorption efficiency, $\eta$, as the only quantity in the right-hand sides of (1) and (2) that depends on the dynamic properties of the antenna. It is an all spectrum measure of the absorption and scattering properties of the object, that is defined by

$$
\eta=\frac{\int_{0}^{\infty} \sigma_{\mathrm{a}}(k) / k^{2} \mathrm{~d} k}{\int_{0}^{\infty} \sigma_{\text {ext }}(k) / k^{2} \mathrm{~d} k}=\frac{\int_{0}^{\infty} \sigma_{\mathrm{a}}(2 \pi / \lambda) \mathrm{d} \lambda}{\int_{0}^{\infty} \sigma_{\text {ext }}(2 \pi / \lambda) \mathrm{d} \lambda},
$$

where $\sigma_{\text {ext }}=\sigma_{\mathrm{a}}+\sigma_{\mathrm{s}}, \sigma_{\mathrm{a}}$, and $\sigma_{\mathrm{s}}$ denote the extinction, absorption, and scattering cross sections, respectively, and $\lambda=2 \pi / k$ is the wavelength. It is clear that $0 \leq \eta<1$ for all objects as $\sigma_{\mathrm{a}} \geq 0$ and $\sigma_{\mathrm{s}} \geq 0$. In $[3,4]$, it is observed that $\eta \approx 1 / 2$ for many small antennas that are matched at a dominant first resonance $k_{0}$. This is partly explained by the fact that the absorption efficiency $\sigma_{\mathrm{a}}\left(k_{0}\right) / \sigma_{\text {ext }}\left(k_{0}\right)=1 / 2$ for minimum scattering antennas, that is, small single mode antennas absorb and scatter the same amount of power at the resonance frequency $[7,8]$. The weighting factor, $k^{-2}$, in (3) emphasizes the dynamics of the antenna for low wavenumbers. Thus, the lower the resonance frequency, the closer $\eta$ is to $1 / 2$ as the resonance region will dominate the integrals. As a consequence, the theory derived here is useful if the analyzed resonance has the lowest frequency. The contributions to $\eta$ in (3) away from the resonance are small due to the fact that scattering dominates the behavior of the antenna in the regions where the mismatch is high.

Here, the case with an idealized lossless antenna that radiates an electric dipole mode is considered to explicitly determine $\eta$ and illustrate how $\sigma_{\text {ext }}(k)$ and $\sigma_{\mathrm{a}}(k)$ depend on the wavenumber around the resonance. A spherical dipole mode at the radius $a$ has the impedance $[6] Z_{\mathrm{TM}}=1 /(\mathrm{j} \omega C)+$ $\mathrm{j} \omega L /\left(1+\mathrm{j} \omega L / \eta_{0}\right)$, where $L=\mu_{0} a, C=\epsilon_{0} a, \omega=k c_{0}$, the time convention $\mathrm{e}^{\mathrm{j} \omega t}$ is used, and $\epsilon_{0}, \mu_{0}, c_{0}$, and $\eta_{0}$ denote the free space permittivity, permeability, speed of light, and impedance, respectively. The impedance is modified by the antenna. We consider an antenna with the input impedance obtained from the impedance of the dipole mode, $Z_{\mathrm{TM}}$, tuned to be resonant at $\omega=\omega_{0}$ with a lumped inductance $L_{1}$, that is,

$$
Z(\omega)=j \omega L_{1}+\frac{1}{j \omega C}+\frac{j \omega L}{1+j \omega L / R_{1}} .
$$

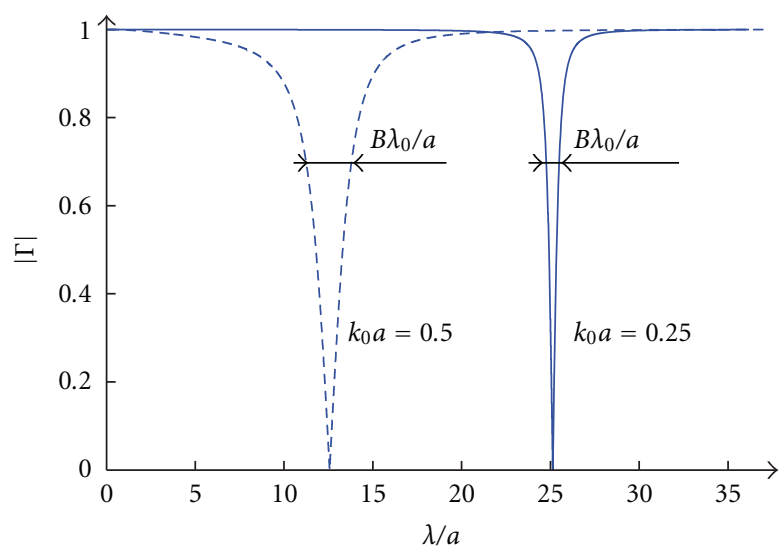

Figure 1: The reflection coefficient of the idealized dipole antenna (4) for $k_{0} a=1 / 2$ and $k_{0} a=1 / 4$ with $C=\epsilon_{0} a, L=\mu_{0} a$ and $R_{1}=\eta_{0}$ as function of the normalized wavelength $\lambda / a=2 \pi c_{0} /(\omega a)$.

The inductance $L_{1}$ is given by $L_{1}=1 /\left(\omega_{0}^{2} C\right)-L /(1+$ $\left.\omega_{0}^{2} L^{2} / \eta_{0}^{2}\right)$, and the radiation resistance at the resonance frequency is $R_{0}=Z\left(\omega_{0}\right)=\omega_{0}^{2} L^{2} R_{1} /\left(R_{1}^{2}+\omega_{0}^{2} L^{2}\right)$, and $R_{1}=$ $\eta_{0}, L=\mu_{0} a$, and $C=\epsilon_{0} a$ in the idealized dipole case. The corresponding $Q$-factor at $\omega=\omega_{0}$ is determined to $Q=1 /\left(C^{2} L R_{1} \omega_{0}^{3}\right)+R_{1} /\left(L \omega_{0}\right)$ and the reflection coefficient, $\Gamma(\omega)=\left(Z(\omega)-R_{0}\right) /\left(Z(\omega)+R_{0}\right)$ has a single resonance with $\Gamma\left(\omega_{0}\right)=0$, see Figure 1 . The absorption cross section (or effective antenna aperture) for lossless antennas is given by [9]

$$
\sigma_{\mathrm{a}}(k)=\frac{D(k)\left(1-\left|\Gamma\left(k c_{0}\right)\right|^{2}\right) \pi}{k^{2}}
$$

where $D(k)=3 / 2$ in the horizontal plane for the considered dipole mode.

Evaluation of $\eta$ in (3) requires a model of the extinction cross section, $\sigma_{\text {ext }}(k)$, that is consistent with (4). Consider a single port antenna with incoming signal $u$ and outgoing signal $v$. The electromagnetic field is expanded in incoming and outgoing spherical modes with coefficients $\mathbf{a}$ and $\mathbf{b}$, respectively. This gives the scattering matrix [10]

$$
\left(\begin{array}{ll}
\Gamma & \mathbf{R} \\
\mathbf{T} & \mathbf{S}
\end{array}\right)\left(\begin{array}{l}
u \\
\mathbf{a}
\end{array}\right)=\left(\begin{array}{l}
v \\
\mathbf{b}
\end{array}\right)
$$

where $\Gamma$ is the reflection coefficient, $\mathbf{R}$ is an $1 \times \infty$ matrix with elements $R_{\mathrm{n}}$, T is an $\infty \times 1$ matrix with elements $T_{\mathrm{n}}$, and $\mathbf{S}$ is an $\infty \times \infty$ matrix with elements $S_{\mathrm{mn}}$.

For simplicity, order the modes such that the idealized dipole antenna radiates the first mode, that is, $R_{\mathrm{n}}=T_{\mathrm{n}}=0$ for $n>1$. Conservation of energy shows that the amplitudes of the reflection coefficient and the scattering coefficient, $S_{11}$, are identical in this case, that is,

$$
\left|\Gamma\left(k c_{0}\right)\right|=\left|S_{11}(k)\right|
$$

for $k \in \mathbb{R}$. Moreover, the scattering matrix is noncausal, that is, it increases as $\mathrm{e}^{2 \mathrm{j} k a}$ as $k \rightarrow \infty$ with $|\arg (\mathrm{j} k)|<\pi / 2-\alpha$, for some $\alpha>0$, where $a$ denotes the radius of the smallest 
circumscribing sphere, see $[11,12]$. As the amplitude of $S_{11}$ is determined by the reflection coefficient (7) they can only differ by a function that has unit magnitude for $k \in \mathbb{R}$. Using rational functions, that is, Blaschke products [12], gives the model

$$
S_{11}(k)=\mathrm{e}^{2 \mathrm{j} k a} \frac{Z\left(k c_{0}\right)-R_{0}}{Z\left(k c_{0}\right)+R_{0}} \prod_{\mathrm{n}} \frac{k_{\mathrm{n}}-k}{k_{\mathrm{n}}^{*}-k}
$$

where $k_{\mathrm{n}}$ denote the zeros of $S_{11}$ in $\operatorname{Re}\{j k\}>0$.

The extinction cross section is often expressed in the transition matrix. It is related to the $S$-matrix in (6), $S$, via $\mathcal{T}_{\mathrm{mn}}=\left(S_{\mathrm{mn}}-1\right) / 2$. Consider an idealized dipole antenna that is resonant for $k_{0} a \ll 1$. The scattering from higherorder modes is negligible for $k_{0} a \ll 1$ so the extinction cross section is well approximated with the dipole mode in this region. The extinction cross section from the dipole mode is hence approximated by

$$
\sigma_{\mathrm{ext}}(k) \approx-\frac{6 \pi \operatorname{Re}\left\{\mathcal{T}_{11}(k)\right\}}{k^{2}}, \quad \sigma_{\mathrm{s}}(k) \approx \frac{6 \pi\left|\mathcal{T}_{11}(k)\right|^{2}}{k^{2}},
$$

where $\mathcal{T}_{11}$ denotes the diagonal dipole element of the transition matrix [12]. Consider the simplest possible case with a single zero $k_{1}$. The value of $k_{1}$ is determined by (8) and (9) inserted into the low-frequency expansions [13] $\sigma_{\text {ext }}(k)=\mathcal{O}\left(k^{2}\right)$ and $\sigma_{\mathrm{s}}(k)=\mathcal{O}\left(k^{4}\right)$ as $k \rightarrow 0$. This shows that $k_{1}=\mathrm{j} /\left(a-C R_{0} c_{0}\right)$, giving the model

$$
S_{11}(k)=\mathrm{e}^{2 \mathrm{j} k a} \frac{Z\left(k c_{0}\right)-R_{0}}{Z\left(k c_{0}\right)+R_{0}} \frac{1-\mathrm{j} k\left(a-C R_{0} c_{0}\right)}{1+\mathrm{j} k\left(a-C R_{0} c_{0}\right)} .
$$

The cross sections $\sigma_{\text {ext }}, \sigma_{\mathrm{a}}$, and $\sigma_{\mathrm{s}}$ are depicted in Figure 2 for the same cases as in Figure 1. It is observed that the areas under the curves are concentrated to the resonances and that $\sigma_{\mathrm{a}}(k) \approx \sigma_{\mathrm{s}}(k)$ around the resonances for $k_{0} a=0.25$. For minimum scattering, $\operatorname{Re}\left\{\mathcal{T}_{11}(k)\right\}=-1 / 2$ in (9), we obtain the envelope $\sigma_{\text {ext }}(k) / a^{2} \approx 3 \pi / k^{2} a^{2}=3 \lambda^{2} / 4 \pi a^{2}$, also plotted in Figure 2. The more dominant a resonance, the closer the obtained value of the extinction cross section is to this envelope at the resonance frequency.

The generalized (all spectrum) absorption efficiency (3) for the idealized dipole is finally determined by

$$
\eta=\frac{\int_{0}^{\infty}\left(\left(1-|\Gamma(k)|^{2}\right) D \pi / k^{4}\right) \mathrm{d} k}{\int_{0}^{\infty}\left(\sigma_{\mathrm{ext}}(k) / k^{2}\right) \mathrm{d} k} \approx \frac{\int_{0}^{\infty}\left(1-|\Gamma(k)|^{2} / k^{4}\right) \mathrm{d} k}{-4 \int_{0}^{\infty}\left(\operatorname{Re}\left\{\mathcal{T}_{11}(k)\right\} / k^{4}\right) \mathrm{d} k} .
$$

The generalized absorption efficiency $\eta$ is evaluated for the idealized dipole model (4) and (10) as well as various other parameter values on $C, L$, and $R_{1}$ in Figure 3 . It is observed that $\eta \lesssim 1 / 2$ for $k_{0} a \ll 1$. The deviation from $1 / 2$ is due to the region with small $\sigma_{\mathrm{s}}$ but negligible $\sigma_{\mathrm{a}}$ for $\lambda / a<8$ as seen in Figure 2.

The particular impedance (4) is not crucial for this result. It is sufficient that the contributions to the integrals in (3) are dominated by the region around the resonance $k_{0}$ and that $\sigma_{\mathrm{a}}$ and $\sigma_{\text {ext }}$ have similar bandwidths and shapes. It is common to assume antennas with a single resonance structure [14] to relate the bandwidth with the antenna $Q$.

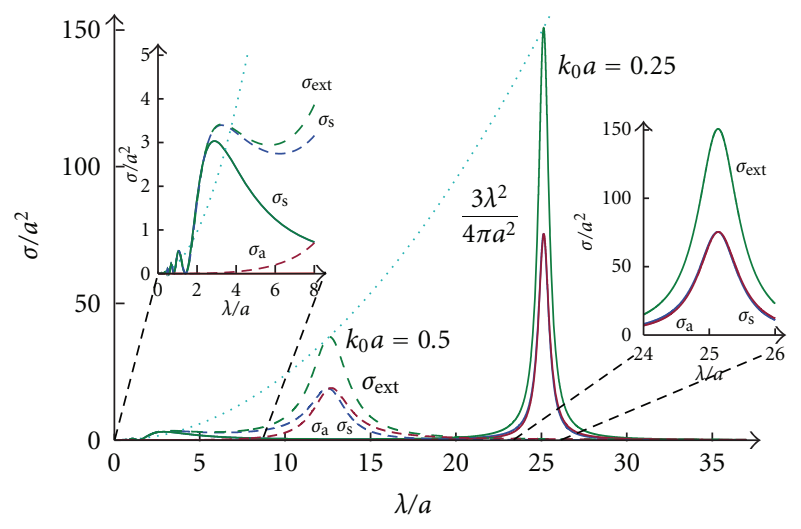

FIgure 2: The extinction, $\sigma_{\text {ext }}$, absorption, $\sigma_{\mathrm{a}}$, and scattering, $\sigma_{\mathrm{s}}$, cross sections for the idealized dipole antenna (4), depicted in Figure 1, and (10) as function of the normalized wavelength $\lambda / a=$ $2 \pi /(k a)$.

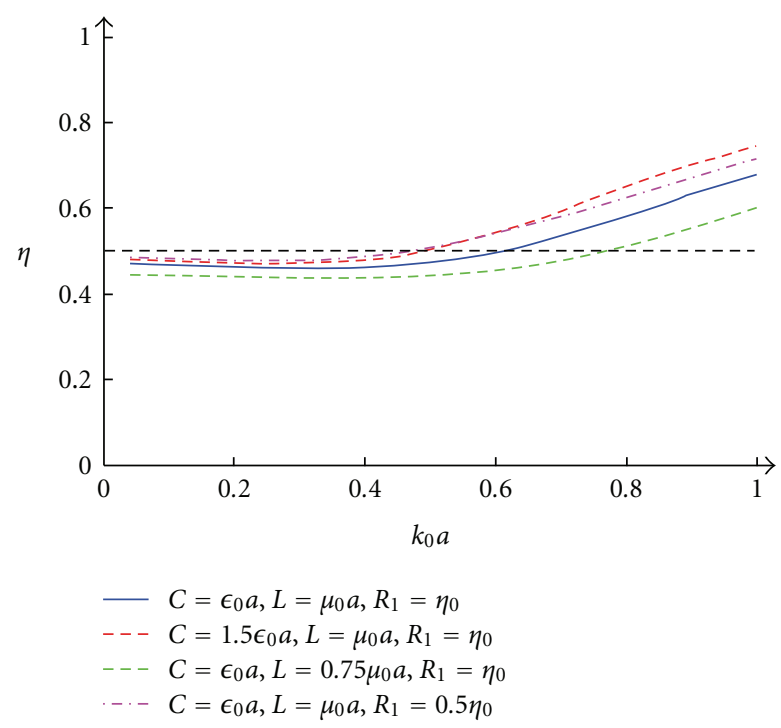

FIGURE 3: The generalized absorption efficiency for the idealized dipole antenna (4) and (10) and various values of $C, L$, and $R_{1}$ as function of the normalized resonance wavenumber $k_{0} a$.

Similarly, assume that the transition matrix element $\mathcal{T}_{11}(k)$ has a single resonance at $k_{0}$ and is minimum scattering, that is, $S_{11}\left(k_{0}\right)=0$ implying $\mathcal{T}_{11}\left(k_{0}\right)=-1 / 2$. The resonance model has complex valued poles at $k \approx \pm k_{0}$ and the shape of the classical Lorentz or resonance circuit $[12,14]$ around the resonance, that is,

$$
\widetilde{T}_{11}(k)=\frac{-1 / 2}{1+(\mathrm{j} / \nu)\left(k / k_{0}-k_{0} / k\right)}=\frac{-\mathrm{j} v k /\left(2 k_{0}\right)}{1-k^{2} / k_{0}^{2}+\mathrm{j} v k / k_{0}},
$$

with $0<v \ll 1$. It satisfies

$$
-\operatorname{Re}\left\{\mathcal{T}_{11}(k)\right\}=\frac{1 / 2}{1+\left(1 / \nu^{2}\right)\left(k / k_{0}-k_{0} / k\right)^{2}}=2\left|\mathcal{T}_{11}(k)\right|^{2},
$$




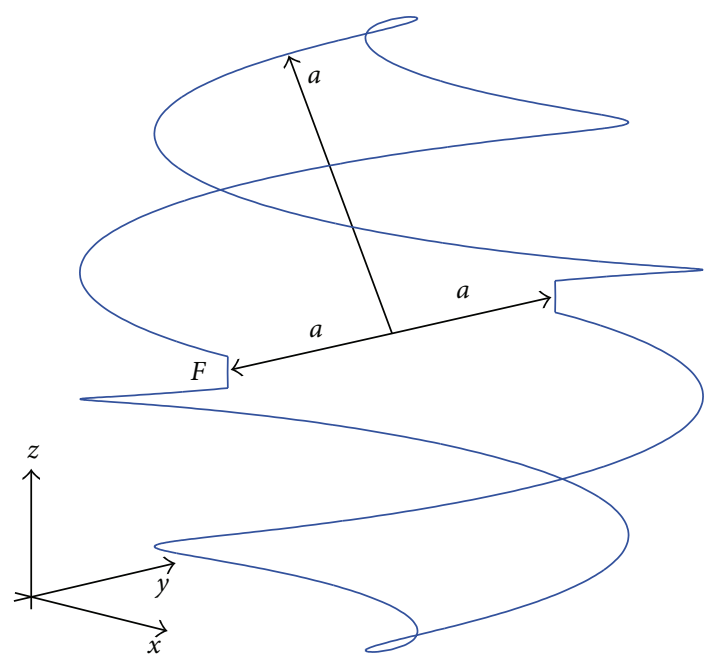

FIgURE 4: Geometry of the two arm spherical helix with circumscribing sphere radius $a=62 \mathrm{~mm}$ and wire radius $R_{\mathrm{w}}=2 \mathrm{~mm}$.

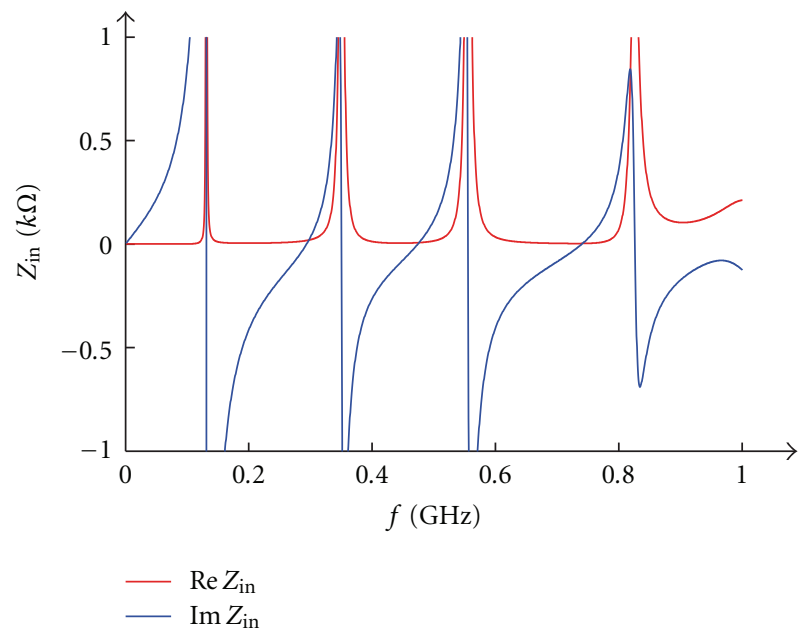

FIgURE 5: Input impedance of the spherical helix depicted in Figure 4 .

for all $k \in \mathbb{R}$ showing that $\sigma_{\text {ext }}(k)=2 \sigma_{\mathrm{s}}(k)=2 \sigma_{\mathrm{a}}(k)$ around the resonance wavenumber; see the $k_{0} a=1 / 4$ case in Figure 2. For antennas with negligible $\sigma_{\mathrm{a}}$ away from the resonance, for example, the dipole model (4), the $\sigma_{\text {ext }} \approx \sigma_{\mathrm{s}}$ contribution to the integral (3) away from the resonance gives $\eta \lesssim 1 / 2$.

\section{Numerical Examples}

The above theoretical results have been analyzed for a number of geometries by numerical simulations; the numerical results show very good agreement with the theory. For each of the examples the approach was the same; first we started with the design and simulation of a radiating structure using the Method of Moments (MoM) simulator in Efield. (www.efieldsolutions.com) Then the radiation resistance at the first resonance was used as a load at the feeding point in a forward scattering simulation, performed using the

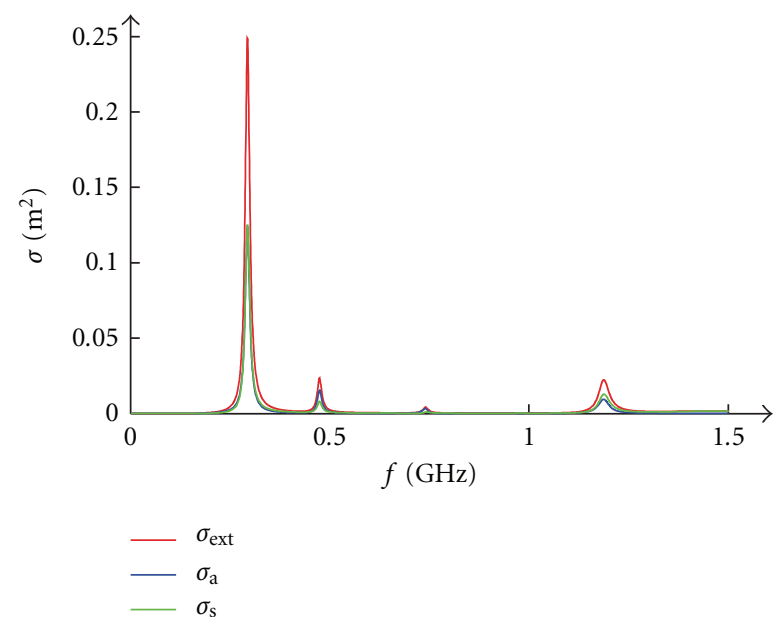

FIGURE 6: Extinction, absorption, and scattering cross sections of the spherical helix depicted in Figure 4.

same software. The results of the antenna and scattering simulations have been used to numerically compute the theoretical parameters using Matlab. Note that not all the available digits are presented in the text as the numerical accuracy does not justify them. However, the formulas are computed without truncation.

3.1. Folded Spherical Helix-D $-1.5, k_{0} a=0.38$. We first describe the results for the folded spherical helix [15] depicted in Figure 4. It comprises a closed loop of perfectly electric conducting wire of radius $R_{\mathrm{W}}=2 \mathrm{~mm}$ that is folded on the surface of a sphere of radius $58 \mathrm{~mm}$ thus obtaining a structure with the radius of the smallest circumscribing sphere $a=62 \mathrm{~mm}$. The structure has two arms of equal length (approximately $l_{\mathrm{a}}=646 \mathrm{~mm}$ ) symmetric with respect to the $z$ axis.

The first step in the analysis is to simulate this structure with Efield. The antenna parameters are determined with an ideal voltage source connected at point $F$ (see Figure 4 ). The resulting input impedance is plotted in Figure 5. The first interesting resonance from a practical point of view appears around $294 \mathrm{MHz}$ with a radiation resistance of $17 \Omega$. It is this resonance that is used to illustrate the physical bounds in [1-4]. At this frequency the antenna radiates a $z$ dipole type pattern. First we compute the $D /\left(Q k_{0}^{3} a^{3}\right)$ value using the simulation data from Efield and the method proposed in $[14,16]$ to approximate the $Q$ factor. The computed values $D=1.5, Q=43$, and $k_{0} a=0.38$ result in the quotient $D /\left(Q k_{0}^{3} a^{3}\right)=0.63$.

The second step is to evaluate the right-hand side in (2). Here, the polarizability dyadics reduce to the high-contrast polarizability dyadic of the perfect electric conductor which is computed using a MoM algorithm (see, e.g., [4]) that solves the electrostatic problem associated with the wire geometry. With $\widehat{\mathbf{e}}=\widehat{\mathbf{z}}$ and only high-contrast electric material present $\widehat{\mathbf{e}} \cdot \boldsymbol{\gamma}_{\mathrm{e}} \cdot \widehat{\mathbf{e}}+(\widehat{\mathbf{k}} \times \widehat{\mathbf{e}}) \cdot \boldsymbol{\gamma}_{\mathrm{m}} \cdot(\widehat{\mathbf{k}} \times \widehat{\mathbf{e}})$ evaluates to

$$
\hat{\mathbf{z}} \cdot \boldsymbol{\gamma}_{\infty} \cdot \hat{\mathbf{z}}=2 \cdot 10^{-3} \mathrm{~m}^{3} .
$$




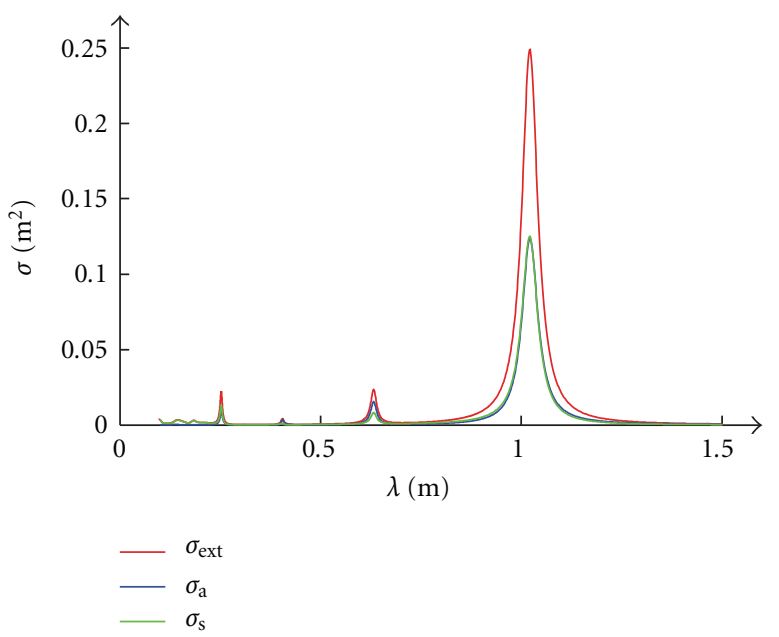

FIGURE 7: Extinction, absorption, and scattering cross sections of the spherical helix depicted in Figure 4 as function of wavelength, $\lambda=2 \pi / k=c_{0} / f$.

The generalized absorption efficiency is computed from the Efield simulation data with (3) and the definitions of absorption and extinction cross sections from [3, 17]. After an integration of 5999 absorption and extinction cross sections samples taken equidistantly between $1 \mathrm{MHz}$ and $3 \mathrm{GHz}$ (see Figure 6 ) we obtain $\eta \approx 0.51$ and write

$$
\frac{D}{Q k_{0}^{3} a^{3}} \approx 0.63 \leq 0.67 \approx \frac{\eta}{2 \pi a^{3}}\left(\hat{\mathbf{z}} \cdot \boldsymbol{y}_{\infty} \cdot \hat{\mathbf{z}}\right) .
$$

This is a true relation showing that the antenna performs close to the $D / Q$ bound of the wire structure.

Moreover, the integrated extinction cross section is related to the polarizability of the structure, as stated in [17] that is,

$$
\frac{2}{\pi} \int_{0}^{\infty} \frac{\sigma_{\text {ext }}(k)}{k^{2}} \mathrm{~d} k=\frac{1}{\pi^{2}} \int_{0}^{\infty} \sigma_{\text {ext }}(\lambda) \mathrm{d} \lambda \approx 1.99 \cdot 10^{-3} \mathrm{~m}^{3},
$$

which is approximately $1 \%$ away from the polarizability determined from the MoM simulation. This deviation can be attributed to the high frequencies (low wavelengths) which are missing in Figure 7. The cross sections should show one dominant resonance and asymptotically tend to 0 for low frequencies.

The physical bounds in (2) create a link between the dynamic properties of the radiating structure and its static properties described by the electric and magnetic polarizability dyadics. Because many common antennas have a generalized absorption efficiency of approximately $1 / 2$ obtaining the bounds for an antenna reduces to a static problem of computing the polarizability dyadics for the geometry, which is easily solved using a Method of Moments algorithm.

It is very important to distinguish the geometry of the radiating structure from its smallest circumscribing sphere. The antenna can be optimized in the limit given by its own polarizability, for example, the smallest circumscribing sphere of the helix in Figure 4 has the radius $a=62 \mathrm{~mm}$,

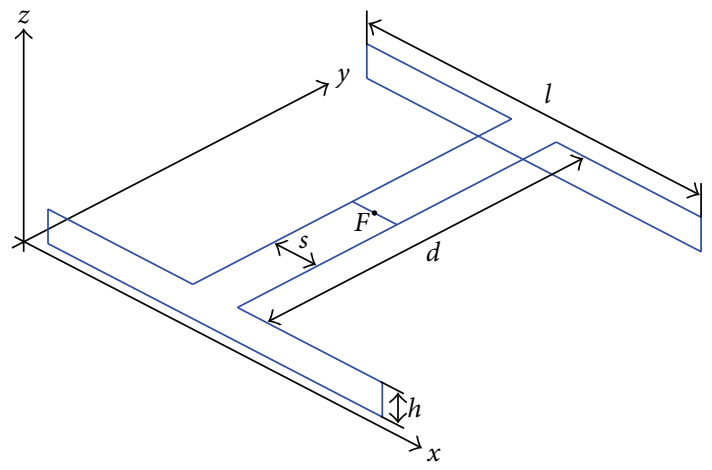

Figure 8: Two-element array of folded dipoles with $l=492.9 \mathrm{~mm}$ and $d=470 \mathrm{~mm}$.

which gives $\hat{\mathbf{z}} \cdot \gamma_{\infty} \cdot \hat{\mathbf{z}}=4 \pi a^{3} \approx 3 \cdot 10^{-3} \mathrm{~m}^{3}$. Thus the maximum attainable value for the $D / Q$ quotient is $D /\left(Q k_{0}^{3} a^{3}\right) \leq 1$ whereas the wire structure simulated here has a maximum attainable value $D /\left(Q k_{0}^{3} a^{3}\right) \leq 0.67$. The presence of the $\left(k_{0} a\right)^{3}$ term allows radiating structures to be directly compared even though they do not have the same size. It can be stated that the wire structure of the helix in Figure 4 can only reach $67 \%$ of the best attainable performance of an antenna circumscribed by a sphere with equal radius. Hence, it is necessary to use a structure with high polarizability to improve the performance, for example, the polarizability of the spherical helix increases with the number of arms and with the wire radius.

3.2. Folded Dipole Array $-D=2.6, k_{0} a=1.7$. The second considered structure is a folded dipole array [18]. The dimensions in Figure 8 are the following: $l=492.9 \mathrm{~mm}$, $d=470 \mathrm{~mm}, h=40 \mathrm{~mm}$, and $s=65.8 \mathrm{~mm}$. The structure is assumed to be fed at point $F$ with an ideal voltage source. All wires have a radius of $R_{\mathrm{w}}=4 \mathrm{~mm}$ thus simulating one possible realization of a simple and common array design using the same type of conductor for all the elements. The smallest circumscribing sphere has the radius $a=347 \mathrm{~mm}$.

First we simulate the structure in transmission in order to obtain the impedance behavior in the frequency range of interest, see Figure 9. The first resonance with practical relevance is close to $233 \mathrm{MHz}$ and, at this frequency the antenna has an input impedance of $59 \Omega$. We shall illustrate the bounds using the characteristics of the structure at this frequency. The far field radiation pattern at this resonance consists of two linearly polarized ( $\hat{\mathbf{x}}$-direction) pencil beams in the broad sides, with a maximum directivity $D=2.6$. The quality factor of this resonance is $Q=4.2$ and with $k_{0} a=1.7$ we obtain the quotient $D /\left(Q k_{0}^{3} a^{3}\right)=0.13$.

The high-contrast polarizability for $\hat{\mathbf{e}}=\hat{\mathbf{x}}$ polarization has the value $\gamma_{\infty, 11}=67.4 \cdot 10^{-3} \mathrm{~m}^{3}$. We note here that the structure is also highly polarizable on the $\hat{\mathbf{y}}$-direction but the $\hat{\mathbf{y}}$-polarization does not contribute to the radiation because of the choice of feeding.

We now turn to the analysis of the cross sections and use 5999 absorption and extinction cross-section samples from equidistantly spaced frequencies between $1 \mathrm{MHz}$ and $3 \mathrm{GHz}$. 
TABLE 1: Numerical results of the antennas in Section 3.3.

\begin{tabular}{|c|c|c|c|c|c|c|c|}
\hline & $D$ & $Q$ & $k_{0} a$ & $D / Q k_{0}^{3} a^{3}$ & $\eta \gamma / 2 \pi a^{3}$ & $\eta$ & $\gamma / a^{3}$ \\
\hline & 1.64 & 8 & 1.51 & 0.056 & 0.058 & 0.51 & 0.705 \\
\hline & 1.64 & 6 & 1.49 & 0.078 & 0.079 & 0.52 & 0.962 \\
\hline & 1.63 & 6 & 1.43 & 0.088 & 0.090 & 0.52 & 1.087 \\
\hline & 1.64 & 3 & 1.44 & 0.173 & 0.155 & 0.50 & 1.944 \\
\hline 官 & 1.55 & 18 & 0.72 & 0.231 & 0.244 & 0.52 & 2.972 \\
\hline 害 & 1.54 & 57 & 0.48 & 0.246 & 0.287 & 0.54 & 3.309 \\
\hline & 2.23 & 5 & 1.31 & 0.211 & 0.200 & 0.52 & 2.429 \\
\hline & 3.04 & 5 & 1.92 & 0.095 & 0.089 & 0.51 & 1.085 \\
\hline & 1.50 & 43 & 0.38 & 0.631 & 0.728 & 0.51 & 9.339 \\
\hline & 2.63 & 4 & 1.69 & 0.130 & 0.130 & 0.48 & 1.698 \\
\hline & 6.15 & 20 & 3.84 & 0.005 & 0.008 & 0.42 & 0.116 \\
\hline & 6.30 & 7 & 4.08 & 0.013 & 0.013 & 0.41 & 0.194 \\
\hline & 3.21 & 17 & 1.72 & 0.036 & 0.042 & 0.14 & 1.897 \\
\hline
\end{tabular}

The first and dominating resonance is shown in Figure 10. The array has a two-band behavior; in either of the two bands it approximately absorbs as much energy as it scatters. Besides these two resonances there is another scattering resonance which contributes to the generalized absorption efficiency. By comparing Figure 6 with Figure 10 we expect to have differences between the two generalized absorption efficiencies but in fact $\eta \approx 0.48$. The reason for the small deviation is the presence of the second resonance very close to the first one, and with comparable values of the radiation resistance.

Gathering the results, we rewrite (2) in numbers as $0.13 \leq 0.13$, thus making this array a structure that is close to the optimal $D / Q$ performance of the wire structure. The perfect matching is explained by the deviation of the structure from the assumed models for the $Q$ factor $(Q \gg 1)$ and the first single and dominant resonance. Compared with a smallest circumscribing sphere, the array only reaches $13 \%$ of its $D / Q$ performance. For evaluating the reliability of the generalized absorption efficiency we integrate the extinction cross section over the wavelength and obtain the value 66.8 . $10^{-3} \mathrm{~m}^{3}$ which is less than $1 \%$ from the previous $\gamma_{\infty, 11}=$ $67.4 \cdot 10^{-3} \mathrm{~m}^{3}$; so the frequency interval is well chosen as to not significantly deviate the resulted $\eta$ from its correct value.

3.3. Other Structures. A number of other structures have been analyzed and the results are gathered in Table 1. The

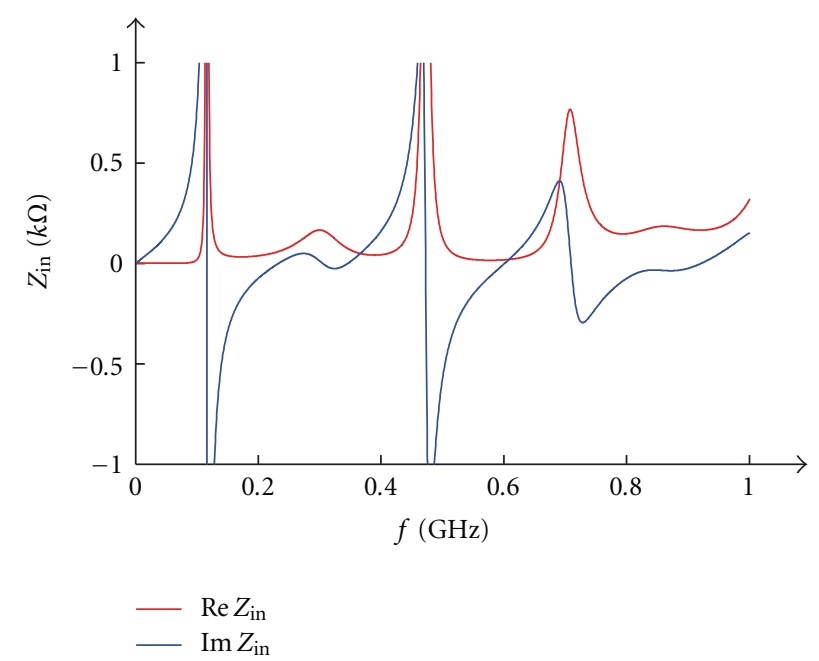

FIGURE 9: Input impedance of the array depicted in Figure 8.

first eight rows in the table correspond to planar geometries circumscribed by different $l_{1} \times l_{2}$ rectangles with $l_{1} / l_{2}$, respectively, equal to: $500,100,25,9,3.6,2,1$, and 0.5 . The polarization is always directed along $l_{1}$. There are two meander-type antennas which differ by their aspect ratios and feeding structure. The ninth and tenth rows correspond 


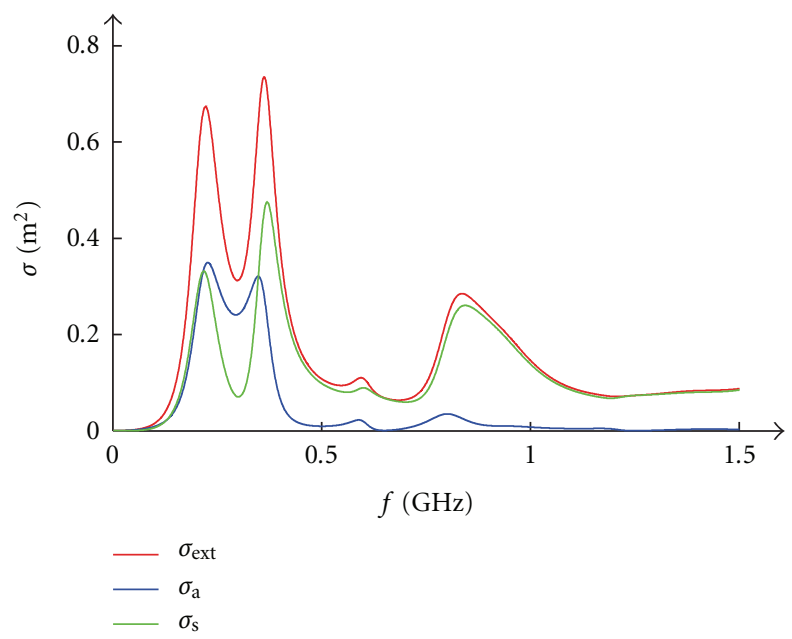

FIgURE 10: Extinction, absorption, and scattering cross sections of the array depicted in Figure 8.

to the structures described, respectively, in Sections 3.1 and 3.2 .

The eleventh and twelfth rows correspond to fourelement arrays fed in phase to obtain two broadside pencilshaped lobes. For the first one the elements are represented by simple dipoles of length $l=500 \mathrm{~mm}$ spaced at $d=$ $500 \mathrm{~mm}$ and wire radius $R_{\mathrm{W}}=1 \mathrm{~mm}$ and for the second the elements are folded dipoles with length $l=502 \mathrm{~mm}$ and height $h=6 \mathrm{~mm}$ spaced at $d=470 \mathrm{~mm}$ and wire radius $R_{\mathrm{w}}=2 \mathrm{~mm}$. Both structures are fed through transmission lines made from the same wire as the radiating elements.

The last line corresponds to a two element array, each element being a Yagi antenna with a reflector $\left(l_{\mathrm{r}}=510 \mathrm{~mm}\right)$, driven element $\left(l_{\mathrm{f}}=500 \mathrm{~mm}\right)$, and director $\left(l_{\mathrm{d}}=420 \mathrm{~mm}\right)$ spaced at $l_{\mathrm{s}}=200 \mathrm{~mm}$. The distance between the elements in the array is $d=470 \mathrm{~mm}$ and the wire radius is $R_{\mathrm{W}}=5 \mathrm{~mm}$. Feeding is realized with a transmission line made from the same wire as the elements.

\section{Conclusions}

We demonstrate that $\eta \lesssim 1 / 2$ for small, $k_{0} a \ll 1$, idealized dipole antennas and for minimum scattering antennas with a dominant first single resonance. As observed in $[3,4]$ this is also valid for several antennas that are of the order $k_{0} a \approx 1$. Here, it is important to realize that the identity (1) is not restricted to electrically small antennas and that $\eta$ in general cannot be replaced by $1 / 2$. Many antennas, for example, YagiUda and reflector antennas have $\eta \ll 1 / 2$ and some, for example, the spiral antenna in [2], have $\eta>1 / 2$.

\section{Acknowledgments}

The financial support by the Swedish Research Council and the Swedish Governmental Agency for Innovation Systems (VINNOVA) within the IMT-advanced and beyond project are gratefully acknowledged. The authors also thank Anders Derneryd for fruitful discussions and comments.

\section{References}

[1] M. Gustafsson, C. Sohl, and G. Kristensson, "Physical limitations on antennas of arbitrary shape," Proceedings of the Royal Society A, vol. 463, no. 2086, pp. 2589-2607, 2007.

[2] C. Sohl and M. Gustafsson, "A priori estimates on the partial realized gain of ultra-wideband (UWB) Antennas," Quarterly Journal of Mechanics and Applied Mathematics, vol. 61, no. 3, pp. 415-430, 2008.

[3] M. Gustafsson, C. Sohl, and G. Kristensson, "Illustrations of new physical bounds on linearly polarized antennas," IEEE Transactions on Antennas and Propagation, vol. 57, no. 5, pp. 1319-1327, 2009.

[4] A. Derneryd, M. Gustafsson, G. Kristensson, and C. Sohl, "Application of gain-bandwidth bounds on loaded dipoles," IET Microwaves, Antennas and Propagation, vol. 3, no. 6, pp. 959-966, 2009.

[5] M. Gustafsson and C. Sohl, "New physical bounds on elliptically polarized antennas," in Proceedings of the 3rd European Conference on Antennas and Propagation (EuCAP '09), pp. 400-402, The Institution of Engineering and Technology, Berlin, Germany, March 2009.

[6] L. J. Chu, "Physical limitations of omni-directional antennas," Journal of Applied Physics, vol. 19, no. 12, pp. 1163-1175, 1948.

[7] J. B. Andersen and A. Frandsen, "Absorption efficiency of receiving antennas," IEEE Transactions on Antennas and Propagation, vol. 53, no. 9, pp. 2843-2849, 2005.

[8] W. Kahn and H. Kurss, "Minimum-scattering antennas," IEEE Transactions on Antennas and Propagation, vol. 13, no. 5, pp. 671-675, 1965.

[9] S. Silver, Microwave Antenna Theory and Design, vol. 12 of Radiation Laboratory Series, McGraw-Hill, New York, NY, USA, 1949.

[10] J. E. Hansen, Ed., Spherical Near-Field Antenna Measurements, IEE Electromagnetic Waves Series, Peter Peregrinus, Stevenage, UK, 1988.

[11] M. Gustafsson, C. Sohl, and S. Nordebo, "Physical bounds on the antenna scattering matrix," in Proceedings of the IEEE International Symposium on Antennas and Propagation, IEEEAP, San Diego, Calif, USA, July 2008.

[12] H. M. Nussenzveig, Causality and Dispersion Relations, Academic Press, London, UK, 1972.

[13] R. E. Kleinman and T. B. A. Senior, "Rayleigh scattering," in Low and High Frequency Asymptotics, V. V. Varadan and V. K. Varadan, Eds., vol. 2 of Handbook on Acoustic, Electromagnetic and Elastic Wave Scattering, chapter 1, pp. 1-70, Elsevier, Amsterdam, The Netherlands, 1986.

[14] M. Gustafsson and S. Nordebo, "Bandwidth, Q factor, and resonance models of antennas," Progress in Electromagnetics Research, vol. 62, pp. 1-20, 2006.

[15] S. R. Best, "The radiation properties of electrically small folded spherical helix antennas," IEEE Transactions on Antennas and Propagation, vol. 52, no. 4, pp. 953-960, 2004.

[16] A. D. Yaghjian and S. R. Best, "Impedance, bandwidth, and $Q$ of antennas," IEEE Transactions on Antennas and Propagation, vol. 53, no. 4, pp. 1298-1324, 2005.

[17] C. Sohl, M. Gustafsson, and G. Kristensson, "Physical limitations on broadband scattering by heterogeneous obstacles," Journal of Physics A, vol. 40, no. 36, pp. 11165-11182, 2007.

[18] G. Hall, et al., The ARRL Antenna Book, American Radio Relay League, 1984. 

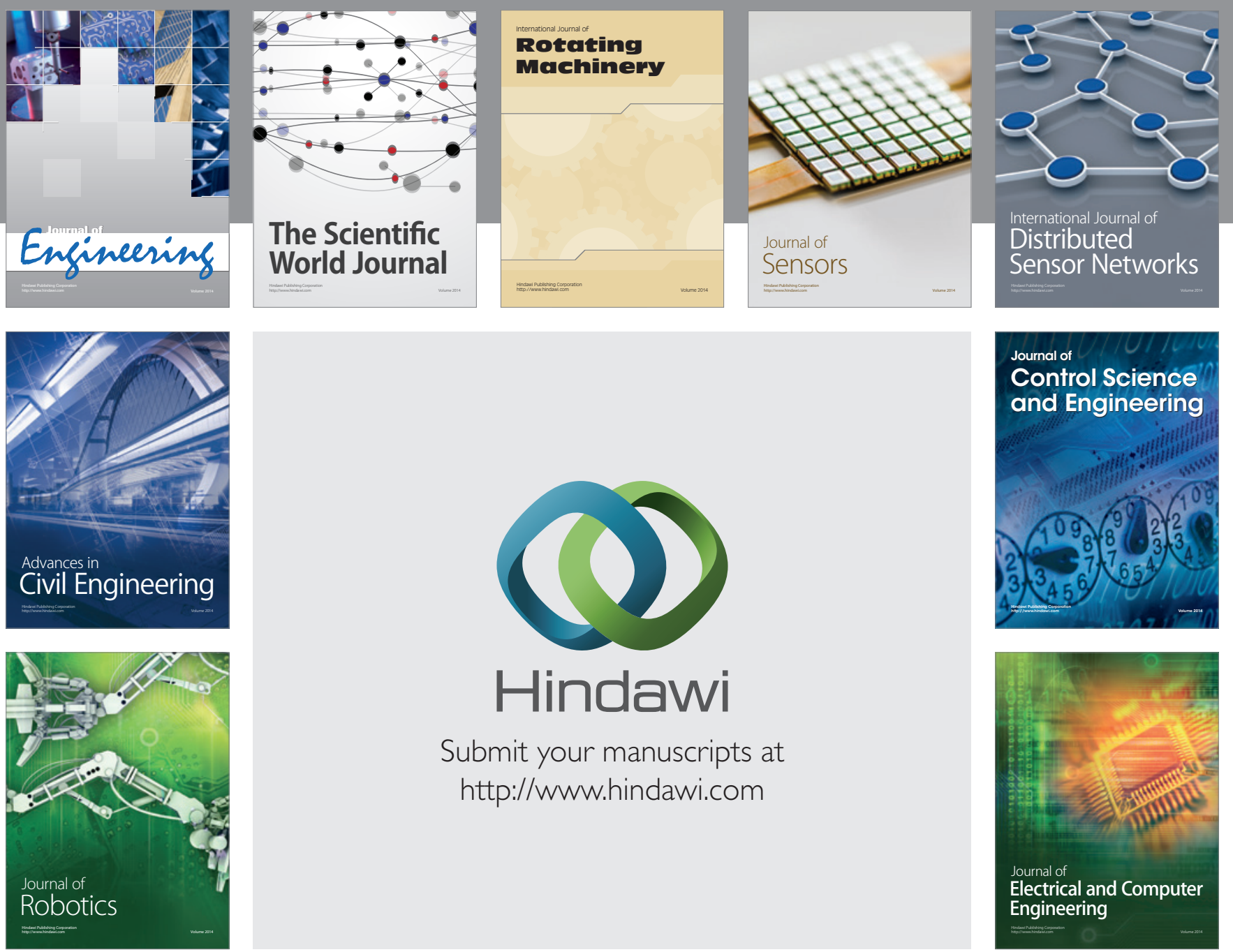

Submit your manuscripts at

http://www.hindawi.com
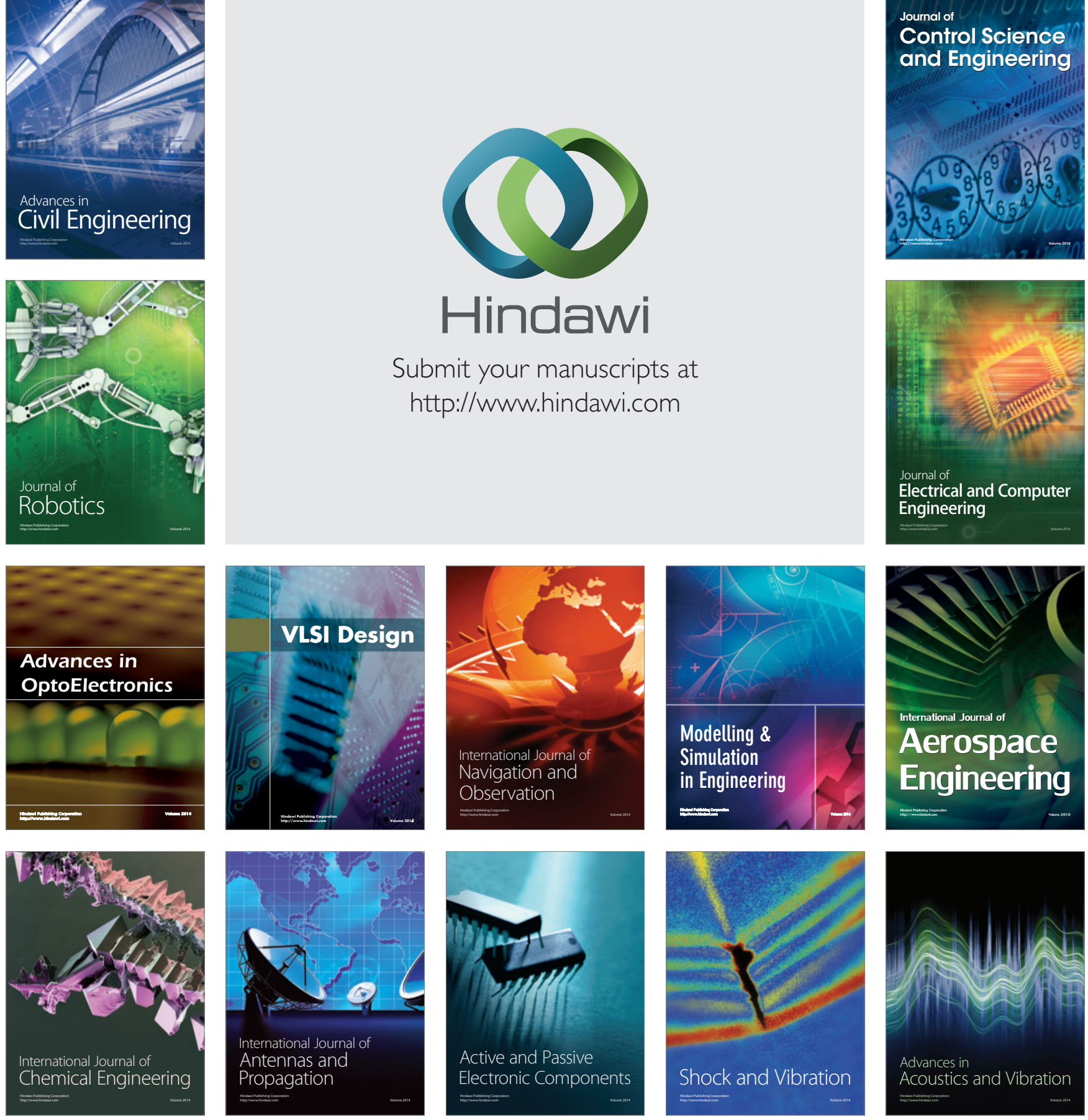